\title{
Abordagem aos Doentes com Intolerância às Estatinas: Revisão Baseada na Evidência
}

\author{
Approach to Patients with Statin Intolerance: \\ Evidence-Based Review
}

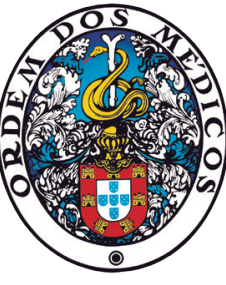

\author{
Joana Tendais ALMEIDA ${ }^{1}$, Ana Luísa ESTEVES $\square^{2}$, Filipa MARTINS ${ }^{3}$, Isabel PALMA ${ }^{4,5}$
}

Acta Med Port 2020 Jan;33(1):49-57 - https://doi.org/10.20344/amp.10376

\section{RESUMO}

Introdução: As estatinas são dos fármacos mais eficazes na redução dos níveis de colesterol e, consequentemente, da morbimortalidade cardiovascular. Apesar de globalmente bem toleradas, têm efeitos secundários que podem condicionar a adesão dos doentes à terapêutica. O objetivo desta revisão é sintetizar a evidência existente acerca da eficácia das estratégias alternativas de abordagem da dislipidemia nos doentes intolerantes às estatinas.

Material e Métodos: Realizámos uma pesquisa bibliográfica de normas de orientação clínica, revisões sistemáticas e meta-análises em janeiro de 2017, nas principais bases de dados internacionais, tendo sido considerados os artigos publicados nos últimos 10 anos e ainda de artigos originais na base de dados PubMed publicados nos últimos três anos. O nível de evidência e força de recomendação foram determinados utilizando a escala de Strenght of Recommendation Taxonomy - SORT.

Resultados: Incluímos oito normas de orientação clínica, seis revisões sistemáticas e um artigo original.

Discussão: A abordagem ao doente intolerante às estatinas varia conforme a gravidade dos sintomas e inclui a manutenção da terapêutica com estatina (redução da dose, introdução de uma estatina de igual ou menor intensidade ou toma em dias alternados), a terapêutica com outros fármacos hipolipemiantes (ezetimiba em monoterapia ou associação com estatina em dose tolerada). A suplementação com coenzima Q10 ou com vitamina D, para melhorar a adesão ao tratamento com estatinas, está desaconselhada. Conclusão: Apesar de apontarmos algumas estratégias de abordagem à intolerância às estatinas, estas baseiam-se maioritariamente em recomendações com evidência fraca a moderada, sendo necessários estudos aleatorizados com maior número de doentes para uma recomendação mais robusta.

Palavras-chave: Dislipidemia/tratamento; Doenças Cardiovasculares/prevenção e controlo; Inibidores de Hidroximetilglutaril-CoA Redutases/efeitos adversos

\section{ABSTRACT}

Introduction: Statins are among the most effective drugs in lowering cholesterol levels and, consequently, in reducing cardiovascular mortality and morbidity. Although generally well tolerated, they have adverse effects that may reduce patient adherence to therapy. The objective of this evidence-based review is to summarize the evidence on the effectiveness of alternative management strategies in patients with intolerance to statins.

Material and Methods: A literature search including clinical practice guidelines, systematic reviews and meta-analyses was conducted, in January 2017, in major international databases, and considered articles published in the last 10 years. The search was complemented with research papers published over the past three years and found in the PubMed database. The level of evidence and strength of recommendation were determined using the scale Strength of Recommendation Taxonomy - SORT.

Results: We included eight guidelines, six systematic reviews and one research paper.

Discussion: The strategies proposed by the different studies vary according to the severity of symptoms of intolerance including maintenance of the statin therapy (dose reduction, addition of a statin of equal or lower intensity or alternate days' uptake) and lipid-lowering therapy with other drugs (ezetimibe monotherapy or association with statin tolerated dose). Supplementation with coenzyme Q10 or vitamin $\mathrm{D}$, in order to improve adherence to treatment with statins, is not recommended.

Conclusion: This review highlights some alternatives to address patients' intolerance to statins; however, these are mostly based on recommendations with low to moderate evidence. Therefore, further research with randomized studies involving greater number of patients is required, in order to obtain a more robust recommendation.

Keywords: Cardiovascular Diseases/prevention \& control; Dyslipidemias/drug therapy; Hydroxymethylglutaryl-CoA Reductase Inhibitors/adverse effects

\section{INTRODUÇÃO}

A doença cardiovascular por aterosclerose dos vasos arteriais é uma das principais causas de mortalidade prematura e perda de anos de vida saudáveis (DALYs - disability-adjusted life years) na Europa. ${ }^{1}$ Sendo a dislipidemia um dos principais fatores de risco cardiovascular

modificáveis, a sua prevenção e tratamento deve fazer parte da abordagem da prevenção cardiovascular, tal como recomendam as guidelines de sociedades europeias e americanas. ${ }^{2,3}$ As estatinas, inibidores da 3-hidroxi-3-methil-glutaril-CoA redutase, reduzem a síntese hepática de

\footnotetext{
1. Departamento de Medicina Geral e Familiar. Unidade de Cuidados de Saúde Personalizados Almeirim. Agrupamento dos Centros de Saúde Lezíria. Santarém. Portugal.

2. Departamento de Medicina Geral e Familiar. Unidade de Cuidados de Saúde Personalizados Vale do Arunca. Agrupamento dos Centros de Saúde do Pinhal Litoral. Leiria. Portugal.

3. Departamento de Medicina Geral e Familiar. Unidade de Saúde Familiar Serpa Pinto. Agrupamento dos Centros de Saúde do Porto Ocidental. Porto. Portugal.

4. Serviço de Endocrinologia. Centro Hospitalar do Porto. Porto. Portugal.

5. Departamento de Endocrinologia. Instituto de Ciências Biomédicas Abel Salazar. Porto. Portugal.

$\square$ Autor correspondente: Ana Luísa Esteves. analuisaestevessp@gmail.com

Recebido: 07 de fevereiro de 2018 - 20 de setembro de 2019 | Copyright @ Ordem dos Médicos 2020
} 
colesterol, sendo dos fármacos mais estudados e eficazes na redução da mortalidade e morbilidade cardiovascular. ${ }^{2,4,5}$ No entanto, apesar de globalmente bem toleradas, as estatinas têm efeitos secundários que podem condicionar a adesão dos doentes à terapêutica, comprometendo assim a sua eficácia no controlo da dislipidemia. ${ }^{6} \mathrm{~A}$ intolerância às estatinas foi definida pelo International Lipid Expert Panel como a incapacidade de tolerar, devido à ocorrência de efeitos secundários, pelo menos, duas estatinas diferentes, uma delas na dose mais baixa, havendo resolução ou melhoria dos sintomas após suspensão do fármaco. ${ }^{6}$ Esta intolerância está relacionada com certos fatores de risco, nomeadamente, a dose de estatina utilizada, o consumo de álcool, idade superior a 80 anos, sexo feminino, história de patologia neuromuscular, renal, hepática e hipotiroidismo. ${ }^{7}$ Os efeitos adversos mais frequentemente associados à terapêutica com estatinas são os sintomas musculares, sendo o termo miopatia usado para descrever o conjunto de todos os sintomas musculares, desde os mais ligeiros como as mialgias e fraqueza muscular, até aos mais graves e raros como a miosite e a rabdomiólise..$^{6,8}$ Apesar de, em ensaios aleatorizados, a incidência de miopatia relacionada com a terapêutica com estatinas ser inferior a $5 \%$, na prática clínica estes sintomas parecem ocorrer em $10 \%$ a $15 \%$ destes doentes, embora menos de $1 \%$ desenvolvam sintomas graves como rabdomiólise. . $^{9,10}$ Considerando esta realidade, há ainda, no entanto, pouca evidência em como abordar a intolerância às estatinas nos doentes com risco cardiovascular. Neste sentido, o objetivo deste trabalho é rever a evidência existente na literatura sobre a eficácia das estratégias alternativas de abordagem da dislipidemia nos doentes intolerantes às estatinas.

\section{MATERIAL E MÉTODOS}

Realizámos uma pesquisa bibliográfica de normas de orientação clínica, revisões sistemáticas e meta-análises em janeiro de 2017, nas bases de dados National Guideline Clearinghouse, National Eletronic Library for Health of British NHS, Canadian Medical Association Practice Guidelines Infobase, Cochrane Library, Database of Abstracts of Reviews of Effectiveness - Centre for Reviews and Dissemination, Bandolier, Evidence Based Medicine Online e PubMed, considerando os artigos publicados nos últimos 10 anos, nas línguas inglesa e portuguesa. Complementámos ainda a pesquisa com os artigos originais na base de dados PubMed, publicados nos últimos três anos. Utilizámos os termos de pesquisa 'statin', uma vez que o termo $\mathrm{MeSH}$ correspondente 'Hydroxymethylglutaryl-CoA Reductase Inhibitors' é pouco utilizado, associado a cada um dos termos MeSH 'myalgia' e 'myopathy' e ainda a cada um dos termos 'intolerance', 'intolerant' e 'rhabdomyolisis'.

Como critérios de inclusão considerámos os estudos cuja população-alvo consistia em doentes com dislipidemia e intolerância às estatinas, sendo esta definida como intolerância a, pelo menos, duas estatinas, cujos sintomas de intolerância desapareceram após a suspensão das mesmas. As abordagens da intolerância às estatinas analisa- das incluíram a manutenção da terapêutica com estatina, a terapêutica com outros fármacos hipolipemiantes e o uso de suplementos para melhorar a adesão à terapêutica com estatinas. Excluímos os artigos cuja intervenção realizada foi a introdução de fármacos ainda não disponíveis em Portugal e/ou aqueles em que a população-alvo incluía doentes com patologia endócrina específica. Como outcome primário foi considerado a ocorrência/recorrência e tolerância a sintomas adversos, nomeadamente mialgias, miosite e rabdomiólise e, secundariamente, analisámos a redução dos níveis de colesterol LDL e da mortalidade, quando disponíveis.

Determinámos o nível de evidência e força de recomendação utilizando a escala de Strenght of Recommendation Taxonomy (SORT) da American Family Physician. ${ }^{11}$

Esta escala classifica os artigos segundo a sua qualidade em três níveis de evidência (nível de evidência 1: estudos com evidência orientada para o doente, de boa qualidade; nível de evidência 2: estudos com evidência orientada para o doente de qualidade limitada; nível de evidência 3: estudos com evidência não orientada para o doente, mas para a doença) e em três graus de força de recomendação (força de recomendação A: evidência orientada para o doente de forma consistente; força de recomendação B: evidência orientada para o doente de forma inconsistente ou qualidade limitada; força de recomendação C: evidência orientada para a doença e baseada em consensos).

A inclusão e a classificação dos artigos segundo a taxonomia SORT foram realizadas pelos três autores revisores de forma independente e determinada através de acordo unânime entre os mesmos.

\section{RESULTADOS}

Na pesquisa inicial identificámos um total de 267 artigos, dos quais obtivemos 240, após remoção dos duplicados. Destes excluímos 150 por não se enquadrarem no objetivo desta revisão. Do total de 90 artigos analisados, 75 foram excluídos por não cumprirem os critérios de inclusão. Assim, após a avaliação dos três revisores, incluímos 15 artigos, dos quais oito normas de orientação clínica, seis revisões sistemáticas e um artigo original, conforme se apresenta na Fig. 1.

\section{Abordagem da intolerância mantendo a terapêutica com estatina}

São várias as abordagens à intolerância às estatinas recomendadas pelas normas de orientação clínica ou alvo de revisões sistemáticas que sugerem a manutenção da terapêutica com estatina numa dose e esquema posológico individualizado. As principais conclusões destes estudos encontram-se resumidas na Tabela 1.

As estratégias propostas nas diferentes guidelines internacionais analisadas variam conforme a gravidade dos sintomas de intolerância. Se estes sintomas forem considerados ligeiros, toleráveis pelo doente ou com níveis de creatina-quinase $(\mathrm{CK})$ inferiores a dez vezes o limite superior do normal, pode ser tentada a redução da dose da 
267 citações identificadas

pela pesquina de base de dados

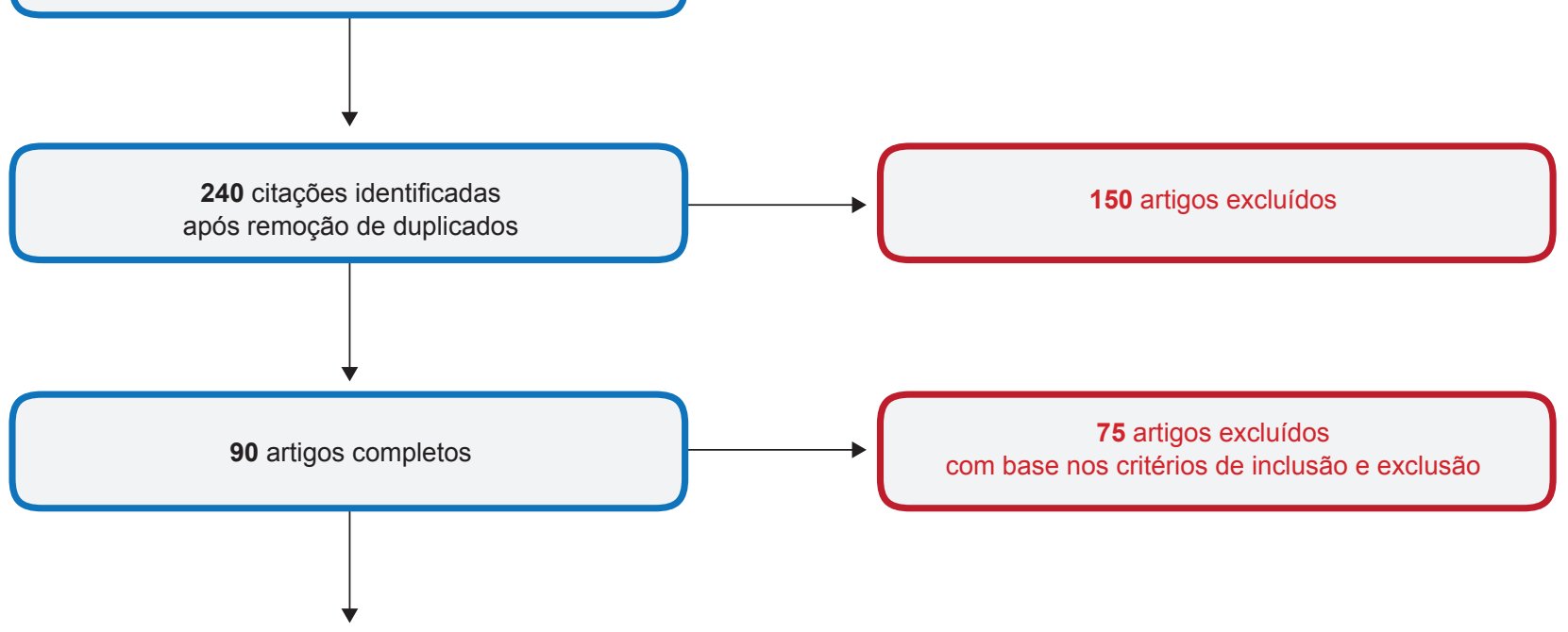

15 artigos incluídos para a revisão:

- 8 NOC;

- 6 revisões sistemáticas;

- 1 artigo original.

Figura 1 - Diagrama de fluxo: resultados

NOC: norma de orientação cínica

estatina previamente utilizada ou a introdução de uma estatina diferente, de igual ou menor intensidade, aumentando a dose até à dose máxima tolerada pelo doente. ${ }^{12-17}$ Como alternativa a estes esquemas é sugerida pelo Canadian Working Group a toma da estatina de maior potência em dias alternados de forma a melhorar a tolerância ao fármaco. ${ }^{16}$ No caso de ser tentada uma reintrodução da estatina a manutenção dos sintomas de intolerância deve ser utilizada como fator de decisão para suspensão ou continuação da terapêutica com a mesma. ${ }^{12,17}$

Se os sintomas de intolerância forem de maior gravidade, a terapêutica com estatina deve ser suspensa até resolução completa da sintomatologia. ${ }^{12,18}$ Posteriormente, segundo a maioria das guidelines analisadas, a mesma estatina ou uma diferente, na mesma dose ou em dose inferior deve ser reintroduzida para testar a reprodutibilidade dos sintomas de intolerância. ${ }^{12-15,17}$

De acordo com a National Guideline Clearinghouse, caso os sintomas musculares ou a elevação da CK se mantenham ao fim de dois meses após a suspensão da estatina devem ser consideradas outras causas para os mesmos. ${ }^{15}$ Se não for comprovada a relação causal entre os sintomas e a terapêutica com estatina, esta deve ser retomada na dose inicial. ${ }^{15}$

Uma alternativa sugerida pela revisão de Jacobson para os doentes que apresentam sintomas musculares induzidos pela toma de estatina é a terapêutica com fluvastatina 80 mg/dia na formulação de libertação prolongada que parece ter sido bem tolerada pelos doentes num ensaio aleatorizado envolvendo 199 doentes com história prévia de sintomas musculares induzidos pela estatina, com um follow-up de 12 semanas. ${ }^{17}$ Se o doente não tolerar esta opção, pode ser considerada a introdução de uma dose reduzida de rosuvastatina ( 5 - $10 \mathrm{mg} / \mathrm{dia}$ ) diária, em dias alternados ou semanalmente, conforme tolerância do doente. ${ }^{17}$

Esta estratégia de toma de estatina de forma não diária foi também avaliada na revisão sistemática de Keating et al, na qual os doentes previamente intolerantes reiniciavam a toma de estatina com atorvastatina ou rosuvastatina apenas numa toma semanal ou bissemanal, sendo esta posteriormente titulada para dias alternados ou três vezes por semana conforme tolerância do doente..$^{19}$ Segundo esta revisão, cerca de $70 \%$ destes doentes foram capazes de tolerar a terapêutica com estatina de forma intermitente, sem recorrência dos efeitos adversos sentidos previamente. ${ }^{19}$ Este estudo demonstrou um controlo variável dos níveis de LDL consoante o esquema utilizado, considerando, desta forma, razoável a diminuição dos níveis de LDL com terapêutica com estatina em esquemas de toma não diária, havendo benefício clínico global. ${ }^{19}$

\section{Terapêutica com outros fármacos hipolipemiantes}

Segundo a maior parte das normas de orientação clínica, perante a recorrência dos sintomas com diferentes estatinas em diferentes doses deve ser introduzida 
Tabela 1 - Normas de orientação clínica e revisões sistemáticas que recomendam abordagem com manutenção da estatina

\begin{tabular}{|c|c|}
\hline $\begin{array}{l}\text { Referêncial } \\
\text { Tipo de Estudo }\end{array}$ & $\begin{array}{l}\text { Recomendações ou } \\
\text { resultados }\end{array}$ \\
\hline $\begin{array}{l}\text { Scottish Intercollegiate } \\
\text { Guidelines Network NHS, } \\
2007^{12}\end{array}$ & $\begin{array}{l}\text { Nos doentes com sintomas ligeiros, pode utilizar-se uma estatina diferente e/ou reduzir a } \\
\text { dose. Com sintomas graves, a estatina deve ser descontinuada. Quando assintomáticos, } \\
\text { pode reintroduzir-se a mesma estatina ou uma diferente em dose igual ou inferior, testando a } \\
\text { reprodutibilidade dos sintomas. }\end{array}$ \\
\hline NOC & $\begin{array}{l}\text { Se a elevação da CK for inferior a } 10 \text { vezes o limite superior do normal, a estatina pode ser } \\
\text { continuada em dose igual ou inferior e os sintomas podem ser marcadores clínicos para a } \\
\text { continuação ou suspensão da estatina. }\end{array}$ \\
\hline $\begin{array}{l}\text { National Guideline } \\
\text { Clearinghouse, } 2013^{15}\end{array}$ & $\begin{array}{l}\text { Se surgirem sintomas musculares ligeiros a moderados: } \\
\text { - A estatina deve ser suspensa até os sintomas serem avaliados; } \\
\text { - Se os sintomas resolverem e não existir contraindicação, deve ser reintroduzida a mesma }\end{array}$ \\
\hline NOC & $\begin{array}{l}\text { estatina em dose igual ou inferior, de forma a estabelecer relação causal; } \\
\text { - Se existir relação causal, a estatina original deve ser descontinuada. Quando os sintomas } \\
\text { resolverem, deve introduzir-se uma estatina diferente em dose baixa; } \\
\text { - Assim que uma dose for tolerada, deve-se aumentar até à dose máxima tolerada. } \\
\text { - Se após dois meses sem estatina os sintomas ou os valores de CK não tiverem normalizado } \\
\text { completamente, devem ser consideradas outras causas; } \\
\text { - Se os sintomas se deverem a outra patologia, retomar a estatina na dose prévia. }\end{array}$ \\
\hline
\end{tabular}

Canadian Working Group Consensus Update, $2013^{16}$

NOC

NICE, $2014^{13,14}$

NOC

European Heart Journal, Joint ESC Guidelines, $2016^{20}$

NOC

Jacobson (2008) ${ }^{17}$

RS

Keating $(2013)^{19}$

RS
Se a reintrodução da mesma estatina não for tolerada, deve ser usada uma dose inferior, uma estatina diferente ou toma em dias alternados.
FR ou

Se surgirem efeitos adversos com estatina em dose elevada, esta pode ser suspensa retomada quando o doente ficar assintomático, ou reduzida a dose até à dose máxima tolerada ou ser substituída por outra de potência semelhante ou inferior.

Os riscos de miopatia podem ser minimizados identificando os doentes com maior probabilidade de intolerância. Pode ser tentada estatina diferente ou em dose baixa várias vezes por semana com aumentos graduais da dose. estatinas (pravastatina, atorvastatina, sinvastatina, lovastatina, fluvastatina).

População estudada: doentes sob terapêutica com estatina

Resultados: se sintomas toleráveis ou níveis de CK inferiores a 10 vezes o limite superior do normal, pode ser mantida a estatina em dose igual ou inferior, sendo os sintomas um guia para a sua manutenção, redução ou suspensão. Se sintomas graves, a estatina deve ser suspensa temporariamente, e se houver resolução dos sintomas, pode ser reiniciada para verificar relação de causalidade.

Uma alternativa é a fluvastatina $80 \mathrm{mg} /$ dia de libertação prolongada. Se não for tolerada, pode ser considerada uma dose reduzida de rosuvastatina (5 - $10 \mathrm{mg} / \mathrm{dia}$ ) diária, em dias alternados ou semanal, conforme tolerância e efeitos adversos.

Variável avaliada: tolerância à reintrodução de estatina em tomas não diárias População estudada: doentes intolerantes à estatina. 10 estudos $(n=575)$

Resultados: cerca de $70 \%$ dos doentes toleraram estatina em toma intermitente, sem recorrência dos efeitos adversos. Parece razoável a diminuição dos níveis de LDL com toma não diária, com benefício clínico global. Recomenda-se atorvastatina ou rosuvastatina semanal ou bissemanal, seguindo-se titulação para dias alternados ou três vezes por semana conforme tolerância.

NOC: norma de orientação clínica; RS: revisão sistemática; FR: força de recomendação; NE: nível de evidência

terapêutica hipolipemiante alternativa, nomeadamente com ezetimiba, fibratos, niacina ou resinas sequestrantes dos ácidos biliares. ${ }^{12,18,20-24}$ Entre estes, de acordo com as guidelines da National Guideline Clearinghouse, o ezetimiba em monoterapia ou em associação com a estatina é recomendado como opção de tratamento na hipercolestero- lemia primária nos adultos intolerantes a estatina ou nos quais a intolerância à estatina impede a sua toma em níveis terapêuticos..$^{20,24}$

As revisões sistemáticas efetuadas por Keating et al, Gudzune et al e Ito et al concluíram que a terapêutica com ezetimiba ou resinas sequestrantes de ácidos biliares em 
monoterapia ou em associação com estatina, nos doentes intolerantes a doses terapêuticas de estatina, pode ser considerada uma alternativa para atingir os valores-alvo de LDL. ${ }^{19,25,26}$ Baseada em evidência cumulativa, esta abordagem parece ter benefícios clínicos e aumentar a tolerabili- dade à estatina. ${ }^{19,25}$ Da mesma forma, a revisão de Jacobson et al conclui que pode ser benéfica a associação de estatina com ezetimiba para atingir os valores-alvo de LDL com menor risco de recorrência de sintomas musculares. ${ }^{17}$

Tabela 2 - Normas de orientação clínica e revisões sistemáticas que recomendam utilização de fármacos hipolipemiantes em alternativa ou em associação à estatina

\begin{tabular}{lll}
$\begin{array}{l}\text { Referência/ } \\
\text { Tipo de Estudo }\end{array}$ & $\begin{array}{l}\text { Recomendações ou } \\
\text { Resultados }\end{array}$ & $\begin{array}{l}\text { FR ou } \\
\text { NE }\end{array}$ \\
\hline $\begin{array}{l}\text { Scottish Intercollegiate } \\
\text { Guidelines Network NHS, } \\
2007^{12}\end{array}$ & $\begin{array}{l}\text { A recorrência dos sintomas com múltiplas estatinas e doses diferentes requer a introdução de } \\
\text { outros agentes hipolipemiantes. }\end{array}$ \\
NOC &
\end{tabular}

Canadian Working Group

Consensus Update,

$2013^{16}$

NOC

NICE, $2014^{14}$

NOC

Dyslipidemia Guideline

Work Group, $2014^{23}$

NOC

National Guideline

Clearinghouse, $2016^{24}$

NOC

European Heart Journal, Joint ESC Guidelines,

$2016^{20}$

NOC

Keating (2013) ${ }^{19}$

RS

Jacobson (2008) $)^{17}$

RS

Gudzune (2014) 25

RS

Ito $(2012)^{26}$

RS
Parece haver segurança e eficácia das bebidas nutracêuticas que contêm niacina, fitosteróis, L-carnitina, vitamina C, coenzima Q-10 e levedura vermelha do arroz.

B

Não há benefício claro em recomendar outro agente hipolipemiante como alternativa à estatina. Doentes com elevado risco cardiovascular que já tiveram um evento cardiovascular devem ser referenciados à Endocrinologia.

Para prevenção primária ou secundária, sugere-se reforço nas alterações do estilo de vida. O tratamento com gemfibrozil ou resinas sequestrantes de ácidos biliares associa-se a ligeira redução do risco cardiovascular em populações limitadas.

A associação de estatina e ezetimiba é recomendada quando não se atingiram os valores-alvo de colesterol total ou LDL.

Os inibidores seletivos da absorção de colesterol (ex.: ezetimiba) não são usados em monoterapia a menos que haja intolerância à estatina.

Os inibidores da PCSK9 diminuem o LDL, quer em monoterapia quer em associação com a dose máxima de estatina.

Variável avaliada: tolerância à reintrodução de estatina em tomas não diárias

População estudada: doentes intolerantes à estatina. 10 estudos $(n=575)$

Resultados: a combinação de estatina com ezetimiba ou resinas sequestrantes de ácidos biliares parece ter benefícios clínicos e aumentar tolerabilidade à estatina.

Variável avaliada: aparecimento de sinais e sintomas musculares (elevação da CK, mialgia, miosite e rabdomiólise) com a toma de diferentes estatinas

População estudada: doentes sob estatina

Resultados: se o doente tolerar uma dose inferior de estatina, mas não suficiente para atingir valor-alvo de LDL, pode ser associada ezetimiba para reduzir risco de recorrência de sintomas. outro agente hipolipemiante

População estudada: doentes com dislipidemia. 36 ECA ( $n>10000)$

Resultados: a associação de estatina de baixa potência com sequestrante de ácido biliar e ezetimiba pode ser alternativa a monoterapia com estatina potente. Apesar de atingidos os níveis-alvo de LDL, não há benefício clínico comprovado na redução do risco cardiovascular.

Variável avaliada: farmacoterapia para redução do risco CV em doentes com dislipidemia População estudada: doentes com dislipidemia.

Resultados: para doentes que não toleram tratamento intensivo com estatina, podem ser considerados outros agentes hipolipemiantes (incluindo ezetimiba e sequestrantes de ácidos biliares) em combinação com estatina. 
No entanto, esta recomendação da utilização de fármacos hipolipemiantes alternativos não é consensual, uma vez que segundo a guideline da NICE 2014 Cardiovascular Risk Assessment and the Modification of Blood Lipids for the Primary and Secondary Prevention of Cardiovascular Disease, não há benefício comprovado com a utilização de outros agentes hipolipemiantes, pelo que nenhuma alternativa às estatinas pode ser recomendada, devendo os doentes intolerantes e com elevado risco cardiovascular ser referenciados para a especialidade de Endocrinologia de modo a serem usadas estratégias alternativas. ${ }^{14}$

As conclusões acima referidas encontram-se resumidas na Tabela 2.

\section{Suplementação para melhorar a adesão ao tratamento com estatinas}

A suplementação com coenzima Q10 ou com vitamina D estão desaconselhadas como estratégia de melhoria de adesão dos doentes à terapêutica com estatina, pelas normas de orientação clínica da NICE e do Canadian Working Group. ${ }^{13,16}$

No mesmo sentido, a revisão sistemática de Marcoff et al não encontrou benefício claro com a suplementação com coenzima Q10 na redução dos sintomas musculares induzidos pela terapêutica com estatina, considerando que a resposta de alguns doentes a esta suplementação se possa dever a um efeito placebo (Tabela 3). ${ }^{27}$

$\mathrm{Na}$ revisão sistemática realizada por Gupta et al, que analisou a suplementação com vitamina $D$ como estratégia de melhoria de tolerância às estatinas, concluiu-se que não há evidência de que esta estratégia diminua os sintomas musculares induzidos pela estatina e que, uma vez mais, a resposta obtida em alguns doentes pode dever-se a efeito placebo. $^{28}$

Apesar de estas revisões apontarem para a não recomendação da suplementação com coenzima Q10, um ensaio clínico mais recente concluiu que esta suplementação reduz os sintomas musculares secundários a terapêutica com estatina e diminui a interferência da dor muscular com as atividades do dia-a-dia. ${ }^{29}$ Por outro lado, o Canadian Working Group sugere que parece haver segurança e eficácia das bebidas nutracêuticas que contêm niacina, fitosteróis, L-carnitina, vitamina C, coenzima Q-10 e levedura vermelha do arroz. ${ }^{16}$

Nesta secção, as principais conclusões encontram-se resumidas na Tabela 3 .

\section{DISCUSSÃO}

A maior parte das guidelines internacionais sugere que a terapêutica da dislipidemia deve, sempre que possível, ser realizada com estatina, em dose e esquemas de toma tolerados pelo doente, ainda que este tenha sido previamente intolerante à estatina e que não sejam atingidos os valores-alvo de LDL recomendados.

Na prática clínica, cerca de $10 \%$ a $15 \%$ dos doentes sob terapêutica com estatina referem sintomas musculares, embora efeitos secundários graves sejam raros. ${ }^{9}$ No entanto apenas uma pequena parte dos sintomas

Tabela 3 - Normas de orientação clínica, revisões sistemáticas e ensaio clínico que recomendam suplementos para melhorar a adesão ao tratamento com estatinas

\begin{tabular}{|c|c|c|}
\hline $\begin{array}{l}\text { Referêncial } \\
\text { Tipo de Estudo }\end{array}$ & $\begin{array}{l}\text { Recomendações ou } \\
\text { resultados }\end{array}$ & $\begin{array}{l}\text { FR ou } \\
\text { NE }\end{array}$ \\
\hline $\begin{array}{l}\text { NICE, } 2014^{13} \\
\text { NOC }\end{array}$ & $\begin{array}{l}\text { Não devem ser prescritas coenzima Q10 ou vitamina D para melhorar a adesão ao } \\
\text { tratamento com estatina. }\end{array}$ & B \\
\hline $\begin{array}{l}\text { Canadian Working Group } \\
\text { Consensus Update, } 2013^{16} \\
\text { NOC }\end{array}$ & $\begin{array}{l}\text { Não estão recomendados suplementos para alívio da mialgia induzida por estatinas. } \\
\text { Não existe associação entre mialgia induzida por estatinas e níveis de vitamina D. A } \\
\text { suplementação com vitamina } D \text { é controversa. }\end{array}$ & B \\
\hline $\begin{array}{l}\text { Marcoff }(2007)^{27} \\
\text { RS }\end{array}$ & $\begin{array}{l}\text { Variável avaliada: níveis de coenzima Q10 em doentes tratados com estatina } \\
\text { População estudada: doentes com dislipidemia. } 22 \text { estudos }(n=1754) \text { : } 10 \text { ECA, } 12 \text { estudos } \\
\text { observacionais } \\
\text { Resultados: sem evidência de que o défice de coenzima Q10 cause miopatia. A } \\
\text { suplementação pode aumentar os níveis de coenzima Q10, mas melhoria dos sintomas } \\
\text { pode ser efeito placebo. }\end{array}$ & 2 \\
\hline $\begin{array}{l}\text { Gupta }(2011)^{28} \\
\text { RS }\end{array}$ & $\begin{array}{l}\text { Variável avaliada: relação entre deficiência de vitamina } D \text { e toma de estatinas } \\
\text { População estudada: doentes com miopatia induzida por estatina } \\
\text { Resultados: sem evidência para a recomendação com vitamina D se não houver défice. } \\
\text { Redução de mialgias pode ser efeito placebo. }\end{array}$ & 2 \\
\hline $\begin{array}{l}\text { Skarlovnik }(2014)^{29} \\
\text { ECA }\end{array}$ & $\begin{array}{l}\text { Variável avaliada: sintomas musculares com a toma de coenzima Q10 } 50 \text { mg duas vezes } \\
\text { por dia vs placebo } \\
\text { População estudada: doentes intolerantes à estatina }(n=50) \\
\text { Resultados: redução dos sintomas musculares e diminuição da interferência da dor } \\
\text { muscular com as atividades do dia-a-dia com a toma de coenzima Q10. }\end{array}$ & 2 \\
\hline
\end{tabular}

NOC: norma de orientação clínica; RS: revisão sistemática; ECA: ensaio clínico aleatorizado; FR: força de recomendação; NE: nível de evidência. 
musculares reportados são, de fato, causados pela terapêutica com estatina. ${ }^{30}$ Nos doentes com mialgia ou miopatia intoleráveis devem ser consideradas e excluídas outras causas de miopatia incluindo hipotiroidismo, atividade física intensa, trauma, abuso de álcool ou drogas e doenças autoimunes (polimiosite, dermatomiosite, polimialgia reumática, entre outras). ${ }^{31,32}$ Pode ainda ser utilizado o questionário Statin Myalgia Clinical Index Score para perceber a probabilidade de os sintomas musculares serem causados pela estatina. ${ }^{33} \mathrm{~A}$ incidência de miopatia varia ainda consoante a estatina utilizada, sendo mais frequente com a terapêutica com atorvastatina (14,9\%) e sinvastatina $(18,2 \%)$ e menos frequentes com fluvastatina $(5,1 \%)$ e pravastatina $(10,9 \%) .{ }^{31,34}$ No entanto, $70 \%$ - $80 \%$ dos doentes que referem sintomas musculares associados a toma da estatina conseguem tolerar a terapêutica. ${ }^{33}$

Como estratégias iniciais de abordagem aos doentes intolerantes à estatina, apresentando sintomas musculares ligeiros e com elevação da CK inferior a 10 vezes o limite superior do normal, destacam-se a substituição da estatina ou a redução da dose inicial da estatina, sendo esta titulada até à dose máxima tolerada pelo doente (nível de evidência 2, força de recomendação B). Neste contexto, a revisão de Jacobson et al sugere, como alternativa, a terapêutica com fluvastatina na formulação de libertação prolongada na dose de $80 \mathrm{mg} / \mathrm{dia}$, a única estatina estudada com boa tolerância por parte dos doentes. ${ }^{17}$

Em caso de manutenção de intolerância com as estratégias iniciais, pode ainda ser considerada a terapêutica com estatina em esquema de toma não diário (nível de evidência 2, força de recomendação B). Na revisão de Keating et al esta estratégia foi considerada com a toma de rosuvastatina ou de atorvastatina semanalmente, duas vezes por semana ou em dias alternados, com tolerância de cerca de $70 \%$ dos doentes estudados e com atingimento dos níveis-alvo de LDL que variam entre $17 \%$ - $84 \%$ consoante os estudos. ${ }^{19}$ Apesar de não haver ensaios aleatorizados de larga escala que testem esta abordagem, nem haver consenso em relação à dose que deve ser utilizada, esta parece uma estratégia com benefício clínico. ${ }^{19} \mathrm{~A}$ escolha da atorvastatina e da rosuvastatina como estatinas preferenciais nestes esquemas de toma não diária prende-se com o facto de serem as estatinas com maior tempo de semivida. ${ }^{31}$

Quando os sintomas de intolerância são de maior gravidade, a terapêutica com estatina deve ser suspensa até resolução completa da sintomatologia (nível de evidência 2, força de recomendação B).

Posteriormente, a mesma estatina ou uma diferente, na mesma dose ou em dose inferior deve ser reintroduzida para testar a reprodutibilidade dos sintomas de intolerância (nível de evidência 2, força de recomendação B).

Caso os sintomas musculares ou a elevação da CK se mantenham ao fim de dois meses após a suspensão da estatina devem ser consideradas outras causas para os mesmos, tal como referido anteriormente, não podendo ser estabelecida uma relação causal e devendo ser retomada a terapêutica com a estatina inicial na dose previamente utilizada. ${ }^{15}$

A recomendação destas estratégias que passam pela tentativa de manutenção da terapêutica com estatina, mesmo em caso de intolerância, deve-se à noção de que a terapêutica com estatina em qualquer dose reduz o risco cardiovascular. ${ }^{13,14}$

No caso de os sintomas de intolerância às estatinas serem graves ou insuportáveis para o doente, as estratégias de abordagem não são consensuais.

Algumas normas de orientação clínica e revisões sistemáticas recomendam a introdução de outros agentes hipolipemiantes como alternativa às estatinas nos doentes intolerantes, de forma a diminuir os níveis de LDL. ${ }^{19,20,24-26}$ Por outro lado, há normas que consideram não existirem alternativas às estatinas por não haver ainda evidência clínica de que a diminuição dos valores de LDL com ezetimiba em monoterapia possa ter benefício clínico. ${ }^{14}$ Uma opção intermédia pode ser considerada na qual a terapêutica seja baseada numa associação de estatina em dose baixa com ezetimiba ou resinas sequestrantes de ácidos biliares, no sentido de atingir os valores-alvo de LDL com menor taxa de efeitos adversos induzidos pela estatina, mas mantendo os benefícios comprovados da terapêutica com a mesma. ${ }^{17}$ Uma vez mais, são necessários mais estudos aleatorizados que possam esclarecer o real benefício clínico cardiovascular dos fármacos alternativos às estatinas, sobretudo em regime de monoterapia.

Em doentes com hipercolesterolemia grave que são intolerantes ou resistentes ao tratamento farmacológico, existem atualmente outras opções de tratamento, onde se inclui a LDL-aférese. O estudo de Palma et al, realizado em Portugal, demonstrou que a técnica de LDL-aférese por adsorção direta de lipoproteínas demonstrou ser um procedimento simples, seguro e eficaz em doentes resistentes ao tratamento nutricional e farmacológico, devendo ser ponderada como tratamento complementar da hipercolesterolemia em doentes intolerantes ou resistentes às estatinas. ${ }^{35}$

$\mathrm{Na}$ pesquisa bibliográfica realizada, foram ainda encontrados ensaios clínicos, geralmente de pequena dimensão, comparando os efeitos de diferentes estatinas com os da levedura vermelha do arroz, de nutracêuticos (levedura vermelha do arroz, berberina, policosanol, ácido fólico, coenzima Q10 e astaxantina), os de Berberis aristata e Silybium marianum e ainda com mipomersen na diminuição dos níveis de LDL, que não foram considerados nesta revisão por não cumprirem os critérios de inclusão no que diz respeito à definição de intolerância às estatinas. ${ }^{36-40} \mathrm{De}$ uma forma geral, estes compostos parecem ser bem tolerados e contribuir para uma diminuição dos níveis de LDL, mas os resultados não são consistentes e os ensaios são de reduzida dimensão.

Existem atualmente novos fármacos a serem considerados como alternativa às estatinas, aprovados pela Agência Europeia do Medicamento (EMA) e a Food and Drug Administration (FDA), nomeadamente os anticorpos monoclonais inibidores da pro-proteína convertase 
subtilisina/kexin (PCSK9), denominados evolocumab e alirocumab. Possuem como alvo a proteína PCSK9, envolvida no controlo da concentração plasmática de LDL, promovendo a redução dos níveis de LDL circulante em cerca de $60 \%$, independente da presença de tratamento hipolipemiante. Dados preliminares de estudos de fase 3 sugerem uma redução de eventos cardiovasculares proporcional à redução de LDL alcançada. São candidatos ao uso destes anticorpos monoclonais os doentes com risco cardiovascular muito elevado, doentes com hipercolesterolémia familiar e doentes intolerantes às estatinas, que mantêm níveis persistentemente elevados de LDL, apesar da dose máxima tolerável da terapêutica de primeira e segunda linha e/ou em aférese. Estes fármacos, administrados por via subcutânea, constituem assim uma alternativa à terapêutica com estatina nos doentes intolerantes. ${ }^{2,41-42}$ No entanto, dada a sua via de administração, encontram-se reservados preferencialmente para os cuidados de saúde hospitalares. Em Portugal, o evolocumab encontra-se disponível, mediante autorização de utilização excecional (QUE) desde 2016. ${ }^{43}$

No que diz respeito à prescrição de suplementos para melhorar a adesão à terapêutica com estatina, a evidência dos estudos disponíveis não permite recomendar a suplementação com coenzima Q10 ou com vitamina $D$ aos doentes com sintomas musculares induzidos pela estatina. Os estudos até agora realizados apontam para um efeito na redução desta sintomatologia semelhante ao placebo.

\section{CONCLUSÃO}

As estratégias propostas pelos diferentes estudos analisados variam conforme a gravidade dos sintomas de intolerância: se estes sintomas forem considerados ligeiros, toleráveis pelo doente, pode ser tentada a redução da dose da estatina previamente utilizada ou a introdução de uma estatina diferente, de igual ou menor intensidade, até à dose máxima tolerada pelo doente. Como alternativa a estes esquemas, é sugerida a toma da estatina de maior potência em dias alternados de forma a melhorar a tolerância ao fármaco. Se os sintomas de intolerância forem de maior gravidade, a terapêutica com estatina deve ser suspensa até resolução completa da sintomatologia. Posteriormente, segundo a maioria das guidelines analisadas, a mesma estatina ou uma diferente, na mesma dose ou em dose inferior deve ser reintroduzida para testar a reprodutibilidade dos sintomas de intolerância. Perante a recorrência dos sintomas adversos com diferentes estatinas em diferentes doses deve ser introduzida terapêutica hipolipemiante alternativa, sendo a ezetimiba o fármaco mais frequentemente recomendado, podendo ser usado em monoterapia ou, preferencialmente em associação com estatina em dose tolerada pelo doente. A suplementação com coenzima Q10 ou com vitamina D como estratégia de melhoria de adesão dos doentes à terapêutica com estatina está desaconselhada.

Os estudos incluídos na presente revisão foram realizados em países desenvolvidos e, por isso, equiparáveis a Portugal. Subsiste a necessidade de mais estudos de boa qualidade, metodologia homogénea e consistente, amostras com dimensão relevante e com maior tempo de follow-up que suportem a evidência de melhoria de outcomes orientados para o doente, nomeadamente a redução do risco cardiovascular, dos fármacos alternativos às estatinas como a ezetimiba, principalmente em monoterapia. Assim, estudos futuros devem avaliar outcomes clínicos a longo prazo e efeitos adversos em doentes intolerantes às estatinas, podendo fornecer informação importante que auxilie a formulação de recomendações para a prática clínica.

\section{CONFLITOS DE INTERESSE}

Os autores declaram não ter conflitos de interesse.

\section{FONTES DE FINANCIAMENTO}

Não foi solicitada qualquer fonte de financiamento.

\section{REFERÊNCIAS}

1. Allender S, Scarborough P, Peto V, Rayner M, Leal J, LuengoFernandez R. European cardiovascular disease statistics. $3^{\text {rd }}$ ed. Brussels: European Heart Network; 2008.

2. Catapano AL, Graham I, De Backer G, Wiklund O, Chapam MJ, Drexel H, et al. 2016 ESC/EAS Guidelines for the management of dyslipidaemias. Eur Heart J. 2016;37:2999-3058.

3. Jellinger PS, Smith DA, Mehta AE, Ganda O, Handelsman Y, Rodbard $\mathrm{HW}$, et al. American Association of Clinical Endocrinologists Guidelines for Management of Dyslipidemia and Prevention of Atherosclerosis. Endocr Pract. 2012;18:S1-78.

4. Brugts JJ, Yetgin T, Hoeks SE, Gotto AM, Shepherd J, Westendorp RG, et al. The benefits of statins in people without established cardiovascular disease but with cardiovascular risk factors: meta-analysis of randomised controlled trials. BMJ. 2009;338:b2376.

5. Cholesterol Treatment Trialists' (CTT) Collaboration. Efficacy and safety of more intensive lowering of LDL cholesterol: a meta-analysis of data from 170000 participants in 26 randomised trials. Lancet. 2010;376:1670-81.

6. Banach M, Rizzo M, Toth PP, Farnier M, Davidson MH, Al-Rasadi K, et al. Statin intolerance - an attempt at a unified definition. Position paper from an International Lipid Expert Panel. Arch Med Sci. 2015;11:1-23.

7. Fitchett $\mathrm{DH}$, Hegele RA, Verma S. Statin intolerance. Circulation. 2015;131:e389-91. statins on skeletal muscle: a perspective for physical therapists. Phys Ther. 2010;90:1530-42.

9. Joy TR, Hegele RA. Narrative review: statin-related myopathy. Ann Intern Med. 2009;150:858-68.

10. Bays H. Statin safety: an overview and assessment of the data-2005. Am J Cardiol. 2006;97:6C-26C.

11. Ebell MH, Siwek J, Weiss BD, Woolf SH, Susman J, Ewigman B, et al. Strenght of Recommendation Taxonomy (SORT): a patient-centered approach to grading evidence in the medical literature. Am Fam Physician. 2004;69:548-56.

12. Scottish Intercollegiate Guidelines Network. Risk estimation and the prevention of cardiovascular disease - a national clinical guideline. Edinburgh: Scottish Intercollegiate Guidelines Network; 2007.

13. National Institute for Health and Care Excellence. Cardiovascular disease: risk assessment and reduction, including lipid modification. 2014. [consultado 2017 jan 14]. Disponível em: https://www.nice.org.uk/ guidance/CG181.

14. National Clinical Guideline Centre. Cardiovascular risk assessment and the modification of blood lipids for the primary and secondary prevention of cardiovascular disease. London: National Institute for Health and Care Excellence; 2014.

15. Stone NJ, Robinson J, Lichtenstein AH, Bairey Merz CN, Blum CB, Eckel
8. Di Stasi SL, MacLeod TD, Winters JD, Binder-Macleod SA. Effects of 
$\mathrm{RH}$, et al. $2013 \mathrm{ACC} / \mathrm{AHA}$ guideline on the treatment of blood cholesterol to reduce atherosclerotic cardiovascular risk in adults: a report of the American College of Cardiology/American Heart Association Task Force on Practice Guidelines. J Am Coll Cardiol. 2014;63:2889-934.

16. Mancini GB, Tashakkor AY, Baker S, Bergeron J, Fitchett D, Frohlich J, et al. Diagnosis, prevention, and management of statin adverse effects and intolerance: Canadian Working Group Consensus Update. Can J Cardiol. 2013;29:1553-68.

17. Jacobson TA. Toward "pain-free" statin prescribing: clinical algorithm for diagnosis and management of myalgia. Mayo Clin Proc. 2008;83:687700 .

18. National Vascular Disease Prevention Alliance. Guidelines for the Management of Absolute Cardiovascular Disease Risk. 2012. [consultado 2017 Jan 14]. Disponível em: https://strokefoundation.org. au/what-we-do/treatment-programs/clinical-guidelines/guidelines-forthe-assessment-and-management-of-absolute-cvd-risk.

19. Keating AJ, Campbell KB, Guyton JR. Intermittent nondaily dosing strategies in patients with orevious statin-induced myopathy. Ann Pharmacother. 2013;47:398-404.

20. European Society of Cardiology. 2016 European Guidelines on Cardiovascular Disease Prevention in Clinical Practice. Eur Heart J. 2016;37:2315-81.

21. All Wales Medicines Strategy Group. Statin template guidance use of statins in primary and secondary prevention of vascular disease. 2012. [consultado 2017 jan 14]. Disponível em: http://www.awmsg.org/ medman_library.html.

22. Meschia JF, Bushnell C, Boden-Albala B, Braun LT, Bravata DM, Chaturvedi S, et al. Guidelines for the primary prevention of stroke: a statement for healthcare professionals from the American Heart Association/American Stroke Association. Stroke. 2014;45:3754-832.

23. Downs JR, O'Malley PG. Management of dyslipidemia for cardiovascular disease risk reduction: synopsis of the 2014 U.S. Department of Veterans Affairs and U.S. Department of Defense Clinical Practice Guideline. Ann Intern Med. 2015;163:291-7.

24. National Institute for Health and Care Excellence. Ezetimibe for treating primary heterozygous-familial and non-familial hypercholesterolaemia. 2016. [consultado 2017 jan 14]. Disponível em: https://www.nice.org.uk/ guidance/ta385.

25. Gudzune KA, Monroe AK, Sharma R, Ranasinghe PD, Chelladurai $Y$, Robinson KA. Effectiveness of combination therapy with statin and another lipid-modifying agent compared with intensified statin monotherapy: a systematic review. Ann Intern Med. 2014;160:468-77.

26. Ito MK. Dyslipidemia: management using optimal lipid-lowering therapy. Ann Pharmacother. 2012;46:1368-81.

27. Thompson ML. The role of coenzyme Q10 in statin-associated myopathy. J Am Coll Cardiol. 2007;49:2231-7.

28. Thompson GA. The relationship of vitamine D deficiency to statin myopathy. Atherosclerosis. 2011;215:21-9.

29. Skarlovnik A, Janić $M$, Lunder $M$, Turk M, Šabovič M. Coenzyme Q10 supplentation decreases statin-related mild-to-moderate muscle symptoms: a randomized clinical study. Med Sci Monit. 2014;20:2183-8.
30. Finegold JA, Manisty $\mathrm{CH}$, Goldacre B, Barron AJ, Francis DP. What proportion of symptomatic side effects in patients taking statins are genuinely caused by he drug? Systematic review of randomised placebo-controlled trials to aid individual patient choice. Eur J Prev Cardiol. 2014;2:464-74.

31. Harper CR, Jacobson TA. Evidence-based management of statin myopathy. Curr Atheroscler Rep. 2010;12:322-30.

32. Chatzizisis YS, Koskinas KC, Misirli G, Vaklavas C, Hatzitolios A Giannoglou GD. Risk factors and drugs interactions predisposing to statin-induced myopathy. Drug Saf. 2010;33:171-87.

33. Taylor BA, Thompson PD. Muscle-related side-effects of statins: from mechanisms to evidence-based solutions. Curr Opin Lipidol. 2015;26:221-7

34. Bruckert E, Hayem G, Dejager S, Yau C, Bégaud B. Mild to moderate muscular symptoms with high-dosage statin therapy in hyperlipidemic patients - The PRIMO Study. Cardiovasc Drugs Ther. 2005;19:403-14.

35. Palma I, Caldas AR, Palma IM, Queirós JA, Madureira A, Oliveira JC. LDL-aférese: experiência do Hospital Santo António. Rev Port Cardiol. 2015;34:163-72.

36. Halbert SC, French B, Gordon RY, Farrar JT, Schmitz K, Morris PB, et al. Tolerability of red yeast rice (2400mg twice daily) versus pravastatin (20mg twice daily) in patients with previous statin intolerance. Am J Cardiol. 2010;105:198-204.

37. Marazzi G, Pelliccia F, Campolongo G, Quattrino S, Cacciotti L, Volterrani $\mathrm{M}$, et al. Usefulness of nutraceuticals (armolipid plus) versus ezetimibe and combination in statin-intolerant patients with dyslipidemia with coronary heart disease. Am J Cardiol. 2015;116:1798-801.

38. Derosa G, Romano D, D'Angelo A, Maffioli P. Berberis aristata combined with Silybum marianum on lipid profilein patients not tolerating statins at high doses. Atherosclerosis. 2014;239:87-92.

39. Visser ME, Wagener G, Baker BF, Geary RS, Donovan JM, Beuers $\mathrm{U}$, et al. Mipomersen, an apolipoprotein B sunthesis inhibitor, lowers low-density lipoprotein cholesterol in high statin-intolerant patients: a randomised, double blind, placebo-controlled trial. Eur Heart J. 2012;33:1142-9.

40. Becker DJ, French B, Morris PB, Silvent E, Gordon RY. Phytosterols, red yeast rice and lifestyle changes instead of statins: a randomized, double-blinded, placebo-controlled trial. Am Heart J. 2013;166:187-96.

41. National Institute for Health and Care Excellence. Evolocumab for treating primary hypercholesterolaemia and mixed dyslipidaemia. 2016 [consultado 2017 jan 14]. Disponível em: https://www.nice.org.uk/ guidance/ta394.

42. National Institute for Health and Care Excellence. Alirocumab for treating primary hypercholesterolaemia and mixed dyslipidaemia. 2016 [consultado 2017 jan 14]. Disponível em: https://www.nice.org.uk/ guidance/ta393.

43. Assessoria de Imprensa do Infarmed, I.P.Acesso à inovação, mais de 1200 doentes tratados com AUE. Infarmed. [consultado 2016 set 9]. Disponível em: http://www.infarmed.pt/documents/15786/1235819/12210347. PDF/9bea0afd-eb8e-43a5-a689-dc3f8e34ae5b. 